\title{
BMJ Open Dietary supplements to reduce symptom severity and duration in people with SARS-CoV-2: study protocol for a randomised, double-blind, placebo controlled clinical trial
}

To cite: Legacy M, Seely D, Conte $\mathrm{E}$, et al. Dietary supplements to reduce symptom severity and duration in people with SARS-CoV-2: study protocol for a randomised, double-blind, placebo controlled clinical trial. BMJ Open 2022;12:e057024. doi:10.1136/ bmjopen-2021-057024

- Prepublication history and additional supplemental material for this paper are available online. To view these files, please visit the journal online (http://dx.doi.org/10.1136/ bmjopen-2021-057024).

DS and KW are joint senior authors.

Received 02 September 2021 Accepted 04 February 2022

Check for updates

(C) Author(s) (or their employer(s)) 2022. Re-use permitted under CC BY-NC. No commercial re-use. See rights and permissions. Published by BMJ.

For numbered affiliations see end of article.

Correspondence to

Dr Dugald Seely;

dseely@thechi.ca

\section{ABSTRACT}

Introduction COVID-19 has caused morbidity, hospitalisations and deaths worldwide. Despite four approved vaccines for COVID-19 in Canada, there is still a need for effective treatments, especially for people in the community. Vaccine efficacy is not $100 \%$ and longterm efficacy is still unknown. Furthermore, there are challenges to herd immunity including vaccine hesitancy and underlying conditions preventing vaccination. We aim to explore if the nutrients vitamin $\mathrm{C}$, vitamin $\mathrm{D}$, vitamin $\mathrm{K}_{2}$ and zinc are an effective treatment option for outpatients diagnosed with COVID-19. The primary outcome is the difference in participant-reported overall health; secondary outcomes include the effect on health status, symptom severity and duration, frequency and length of hospitalisations and mortality.

Methods and analysis This study is a two-arm, parallel-group, double-blind, placebo-controlled, phase III randomised controlled trial. 200 patients will be recruited remotely from COVID-19 test centres in Ottawa, Canada associated with The Ottawa Hospital. Overall health will be measured using the EuroQol Visual Assessment Scale; health status will be measured using the EuroQol 5-dimension 5-level questionnaire; symptom severity and duration will be measured using an independently developed questionnaire; analyses will use an area under the curve approach and compare mean scores using unadjusted $t$ tests. Study data will be recorded on electronic case report forms using the Research Electronic Data Capture platform. An independent data safety and monitoring board will perform ongoing review of the study for feasibility and safety.

Ethics and dissemination This study has received ethical approval from the research ethics boards of the Canadian College of Naturopathic Medicine and the Ottawa Health Sciences Network, as well as regulatory approval from the Therapeutic Products Directorate and Natural and Non-Prescription Health Products Directorate of Health Canada. Results will be published in a peer-reviewed scientific journal with open access.

Trial registration number NCT04780061.
Strengths and limitations of this study

- Blinding, randomisation and placebo control enhance the trial's internal validity.

- Large spectrum of outcomes allows for both rigorous and exploratory analyses.

- The study procedures regarding remote screening, recruitment, follow-up and product dispensation are novel in this field and will act as a framework for future research.

- Virtual nature of the study and necessity for internet literacy may create selection bias.

- Variable length of time from symptom onset to treatment commencement due to delays in result reporting may reduce the effectiveness of the investigational product.

\section{INTRODUCTION}

Background and rationale

COVID-19, caused by the novel SARS-CoV-2, has caused hospitalisations, morbidity and deaths worldwide. COVID-19 causes mild to moderate flu-like symptoms in most people, and can cause severe disease including pneumonia, acute respiratory distress syndrome, multiorgan failure and death particularly in high-risk individuals. ${ }^{1}$ As of June 2021, over 177 million people have been diagnosed with COVID-19 and over 3.8 million deaths have occurred as a result of infection. ${ }^{2}$

There are currently four approved COVID-19 vaccines in Canada. ${ }^{3}$ Despite this, effective treatments are still needed. None of the four vaccines are $100 \%$ effective, and long-term immunity is yet to be determined. ${ }^{4}$ In addition, there are challenges to achieving herd immunity based on an inability for those with certain underlying conditions to be vaccinated and vaccine hesitancy. ${ }^{5}$ Treatments for 
COVID-19 are currently limited. In Canada, the only approved treatments are remdesivir and the monoclonal antibodies bamlanivimab, casirivimab and imdevimab. ${ }^{6}$ Remdesivir is indicated for COVID-19 positive patients with pneumonia needing supplemental oxygen; however, results from trials are mixed. ${ }^{7}$ The three monoclonal antibodies are indicated for people with mild to moderate COVID-19 who are at high risk for progressing to hospitalisation or death. ${ }^{9} 10$ All three monoclonal antibody treatments are permitted under an interim authorisation pending the results of clinical trials to verify their clinical benefit. To date, there are no fully approved treatments for non-hospitalised patients diagnosed with COVID-19.

The use of natural health products (NHPs), including vitamins, minerals and herbs, to treat COVID-19 infections, has received both academic and public attention. ${ }^{11} 12$ Various NHPs have undergone observational and clinical research for a wide variety of other upper respiratory tract infections (URTIs). These trials have mainly focused on the treatment of URTI symptoms, including reducing the duration and severity of the illness. Some of the most heavily researched NHPs include andrographis, ${ }^{13} 14$ quercetin, ${ }^{15}$ vitamin $\mathrm{C},{ }^{16-18}$ vitamin $\mathrm{D}^{19-21}$ and zinc. ${ }^{22}$ At the time of the creation of this protocol (January 2021), there was observational evidence to support some NHPs for the treatment of COVID-19, such as vitamin $\mathrm{D}^{2324}$ and vitamin $\mathrm{K}^{25}$; however, there were no published doubleblind, placebo-controlled, randomised clinical trials studying NHPs and COVID-19 symptoms.

A more recent review of the literature revealed the presence of new COVID-19-specific NHP research. A June 2021 meta-analysis of 10 observational studies and 3 randomised controlled trials (RCT) $(\mathrm{n}=2933)$ reported that supplementation with vitamin D significantly reduced intensive care unit (ICU) admissions and mortality, while reducing the risk of adverse outcomes, exclusively when administered after a COVID-19 diagnosis. ${ }^{26}$ One RCT applying similar interventions as our protocol (8000 mg ascorbic acid and $50 \mathrm{mg}$ zinc gluconate) found a nonstatistically significant benefit for each supplement on their own, and in combination, compared with standard care. $^{27}$ Of note, the study was terminated early due to being underpowered because of recruiting less than half of the intended sample size and experienced considerable compliance issues.

Based on the above studies for COVID-19 and other similar URTIs, the nutrients vitamin $\mathrm{C}$, vitamin $\mathrm{D}_{3}$, vitamin $\mathrm{K}_{2}$ and zinc stood out as the most promising NHPs for the treatment of symptoms caused by COVID-19, and thus were chosen as interventions for this study.

\section{Choice of comparator}

The comparator in this trial will be a placebo. Currently, there are no standard of care treatments for people in the community diagnosed with COVID-19; thus, a placebo offers the most rigorous assessment of efficacy while having no detriment to safety due to lack of standard treatments. Should a participant be hospitalised, they will be asked to stop all study activities, including taking investigational product, until they are back home and able to tolerate the intervention again. If hospital-initiated treatments continue while at home, these will take priority over the study intervention if there is a safety concern identified by the qualified investigator or treating physician. As the investigational products in this study are readily available over the counter, monitoring for contamination and cross-over is imperative. This will be accomplished through weekly follow-up with all participants.

\section{Objectives}

We hypothesise that supplementation with vitamin $\mathrm{D}_{3}$, vitamin $\mathrm{C}$, vitamin $\mathrm{K}_{2}$ and zinc will increase participantreported overall health in outpatients diagnosed with COVID-19 compared with a placebo by reducing the severity and duration of common COVID-19 symptoms experienced in a community setting.

The primary objective of this study is the difference in participant-reported overall health between arms; secondary objectives include:

1. Effect of the intervention on the health status of participants.

2. Symptom severity including self-reported measures for fever, cough, shortness of breath, fatigue, headache, myalgia/arthralgia (body aches), nausea, vomiting, diarrhoea, shakes/chills, congestion and loss of taste and smell.

3. Total symptom duration.

4. Incidence of delayed return to usual health.

5. Frequency of hospitalisations, including emergency room visits, acute care admissions and ICU admissions.

6. Hospital length of stay (if applicable).

7. All-cause mortality.

\section{Study design}

This study is a two-arm, parallel group, double-blind, placebo-controlled, phase III trial powered to detect meaningful differences in the overall health of adults with COVID-19 between the treatment and control arms at 21 days. Total trial duration for participants is 12 weeks, and the treatment period runs for 21 days following randomisation.

\section{PARTICIPANTS, INTERVENTIONS AND OUTCOMES Study setting}

This study will recruit outpatients from Ottawa, Canada from COVID-19 test centres in the community associated with The Ottawa Hospital (TOH). The primary site of study conduct will be the Centre for Health Innovation $(\mathrm{CHI})$, an integrative care clinic located in Ottawa. All recruitment, study activities and follow-up will take place remotely as participants will be in quarantine for at least 14 days. Study staff will only communicate with participants by phone or email, and participants will complete all study activities in their own homes. 


\section{Eligibility criteria}

To be eligible for this study, patients must meet the following inclusion criteria:

1. Adults $(\geq 18)$ who test positive for SARS-CoV-2 by reverse transcriptase PCR in an outpatient setting.

2. Access to internet.

The criterion of access to internet may cause selection bias in our population and will not be entirely representative of COVID-19-positive patients in the Ottawa community; however, due to the nature of the study, the progression of the disease, and the public health recommendations to maintain both safety of participants and research staff, in-person consent and follow-up is not feasible.

In addition, patients must not exhibit any of the following exclusion criteria:

1. Symptom onset greater than 4 days prior to enrolment.

2. Regular supplementation with $>500 \mathrm{mg}$ vitamin $\mathrm{C}$, $>1000$ units vitamin $\mathrm{D}$ (any form), >120 $\mu \mathrm{g}$ vitamin $\mathrm{K}$ (any form) or $>15 \mathrm{mg}$ zinc taken daily within the past month.

3. Currently taking warfarin or an equivalent vitamin $\mathrm{K}$ antagonist anticoagulant.

4. End-stage chronic kidney disease.

5. History of calcium oxalate kidney stones.

6. Active granulomatosis (sarcoidosis, tuberculosis, lymphoma).

7. Known hypercalcaemia or hypervitaminosis D.

8. Currently taking either of the following antibiotics: cephalexin, tetracyclines.

9. Known allergy to any investigational product, silicon dioxide, cellulose or medium chain triglyceride oil.

10. Participating in an investigational study or participation in an investigational study within the past 30 days.

The first exclusion criterion was chosen in an attempt to include patients who have the best chance at a potential benefit from the intervention. The median time to symptom resolution for COVID-19 patients has been reported to be $4-8$ days ${ }^{28}$; therefore, recruitment of patients whose symptom onset date was five or more days before enrolment would likely yield a large proportion of patients whose symptoms have already resolved. The second exclusion criterion balances feasibility with contamination. Those supplementing with one or more of the investigational products could introduce bias to the population and skew results; however, low-dose supplementation is common among Canadians and would severely hinder recruitment.

\section{Interventions}

The interventions for this study will be vitamin C, vitamin $\mathrm{D}_{3}$, vitamin $\mathrm{K}_{2}$ and zinc plus equivalent placebos. All investigational products will be manufactured by New Roots Herbal under the brand name Vitazan Professional. Specific formulations of each investigational product are as follows:
Study product \#1

Vitamin D3 50000 IU.

Formulation: Capsule. Each capsule contains $500 \mathrm{mg}$ (50000 units) cholecalciferol (vitamin $\mathrm{D}_{3}$ ).

Dose: One capsule on day 1 of the intervention period.

Placebo equivalent: microcrystalline cellulose capsule, $350 \mathrm{mg}$.

Absolute contraindications: history of hypervitaminosis D, hypercalcaemia or sarcoidosis.

\section{Study product \#2}

Vitamin $\mathrm{K}_{2} / \mathrm{D} 3$ liquid.

Formulation: Liquid. Each $0.0285 \mathrm{~mL}$ drop contains 30 $\mu \mathrm{g}$ menaquinone-7 (MK-7, vitamin $\mathrm{K}_{2}$ ) and 3.125 $\mu \mathrm{g}(125$ units) cholecalciferol.

Dose: $0.114 \mathrm{~mL}$ (four drops) twice daily for 21 days totalling $240 \mu \mathrm{g}$ MK-7 and 1000 units cholecalciferol per day.

Placebo equivalent: Medium chain triglyceride oil.

Absolute contraindications: history of hypervitaminosis D, hypercalcaemia or sarcoidosis; warfarin or another vitamin $\mathrm{K}$ antagonist anticoagulant.

\section{Study product \#3}

Vitamin C/zinc acetate.

Formulation: Capsule. Each capsule contains $666 \mathrm{mg}$ ascorbic acid (vitamin $\mathrm{C}$ ) and $8.3 \mathrm{mg}$ zinc acetate.

Dose: Three capsules three times daily for 21 days totalling $6 \mathrm{~g}$ ascorbic acid and $75 \mathrm{mg}$ zinc acetate per day.

Placebo equivalent: microcrystalline cellulose capsule, $350 \mathrm{mg}$.

Absolute contraindications: calcium oxalate kidney stones, end-stage chronic kidney disease, cephalexin, tetracycline antibiotics.

Modifications to the intervention schedule are permitted under the discretion of the qualified investigator $(\mathrm{KW})$ or subinvestigator delegated the task. Adherence to the protocol will be participant reported through a phone call at the end of the intervention period. Participants will be asked to conduct pill counts for the two capsule-based products and estimate how many doses they have missed of the liquid product.

Absolute contraindications to the investigational products are outlined above and in the exclusion criteria. Should a participant be placed on one of these medications or be diagnosed with one of the conditions, they will stop taking the study product. There are no rescue medications for this study.

\section{Outcomes, timeline and schedule of events}

The full schedule of events is presented in table 1 . The primary outcome of this study is the difference in mean participant-reported overall health over 21 days between arms measured using the EuroQol Visual Assessment Scale (EQ-VAS) ${ }^{29}$ The EQ-VAS records the respondent's overall current health on a vertical scale between 0 and 100 , where the end points are labelled 'the best health you can imagine' (i.e., a score of 100) and 'the worst 


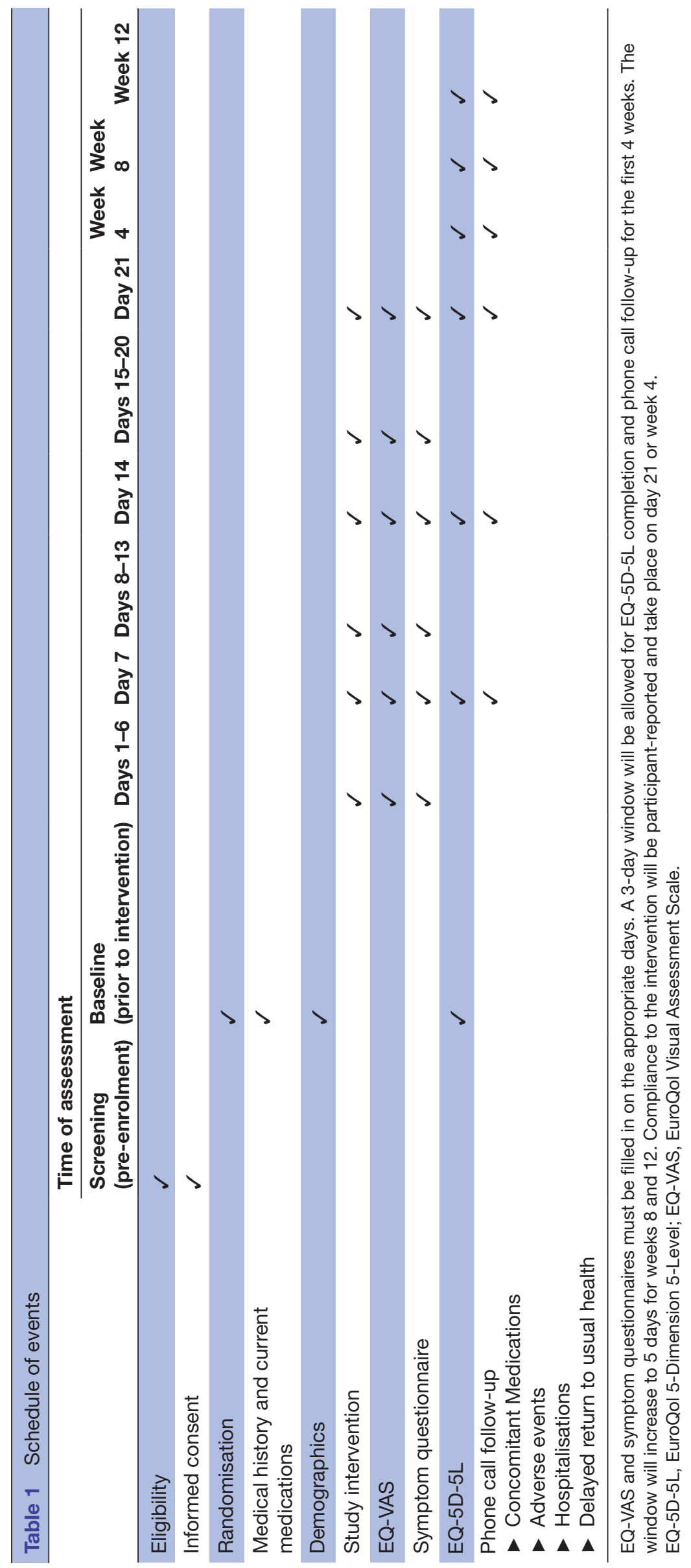

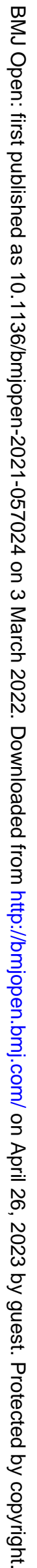


health you can imagine' (i.e., a score of 0 ). The EQ-VAS will be filled out each day while on the intervention (21 days total). The secondary outcomes are as follows:

\section{Health status}

Measured by combining one level from each of the five dimensions of the EuroQol 5-dimension 5-level (EQ-5D-5L) questionnaire ${ }^{29}$ to form a unique health state. The EQ-5D-5L questionnaire will be filled in at baseline and weeks 1, 2, 3, 4, 8 and 12. Mean health state at each time point will be compared between arms.

\section{Symptom severity and duration}

Measured using an internally developed questionnaire specific for the most common COVID-19 symptoms, which include: fever, cough, shortness of breath, fatigue, headache, myalgia/arthralgia (body aches), nausea, vomiting, diarrhoea, chills, altered taste, altered smell and nasal congestion. ${ }^{28}{ }^{30}$ Each symptom will be rated on a 4-point severity scale: 0-none, 1-slight, 2-moderate and 3-severe. Participants will fill in this questionnaire daily while receiving treatment (i.e., for 21 days). We will compare the total mean symptom scores over 21 days between arms.

\section{Incidence of delayed return to usual health}

Measured through follow-up calls with participants at weeks 4,8 and 12 following randomisation. Those experiencing prolonged COVID-19 symptoms lasting 4-12 weeks will be classified as having 'ongoing symptomatic COVID-19,' while those still afflicted at 12 weeks will be classified as having 'post-COVID-19-syndrome. ${ }^{31}$ We will compare the number of participants in each arm who exhibit ongoing symptomatic COVID-19 and postCOVID-19 syndrome.

\section{Hospitalisation}

The rate and type of hospitalisation, as well as the length of stay, will be collected from participant medical records where possible and will otherwise be participant-reported. Information will be collected throughout the 12-week study period.

\section{All-cause mortality}

Date of death will be collected through medical records and obituary searches when medical records are not available. Information will be collected throughout the 12-week study period.

Overall health was chosen as the primary outcome as it uses a validated tool and is easily interpretable. Although research on other URTIs focuses heavily on symptom severity questionnaires, currently there are no validated tools for assessing COVID-19 symptom severity. The clinical significance of symptom severity in a non-validated tool is much more difficult to analyse and open to differing interpretations.

The symptom questionnaire developed by our team is largely based on recommendations from the US Food and Drug Administration guidance document for investigators conducting community clinical trials for COVID-19 prevention or treatment ${ }^{32}$ and commonly reported COVID-19 symptoms in both the hospital and community setting. ${ }^{28} 30$

\section{Sample size}

With respect to the primary outcome of participantreported overall health, power calculations were conducted based on between-group differences and Cohen's guideline for a small effect size of 0.3. A sample size of 176 (88 per arm) provides $80 \%$ power to detect a difference at an alpha of 0.05 . To account for an approximate 10\%-15\% lost to follow-up we will enrol 200 participants (100 per arm).

\section{Recruitment}

Screening for potential participants will be facilitated by clinical staff at COVID-19 test centres in Ottawa associated with TOH. Staff at these test centres are responsible for contacting any person who tests positive for COVID19. The clinical team will use this opportunity to obtain the patient's consent to be contacted by the research team. Nursing staff will notify the trial coordinator of all patients who agree to be contacted. Study staff at the CHI will then contact each patient by phone and further determine their eligibility. If a patient is interested and eligible, they will be taken through the informed consent process and sign an electronic informed consent form using Adobe Sign.

Over time, the COVID-19 case count in Ottawa has varied greatly. With vaccine distribution continuing to increase, we assume the infection rate will remain steady at 10-15 diagnoses per day; however, not all COVID-19 positive patients will be referred to our group due to competing trials and lack of recruitment from all test centres. We expect to receive referrals for $50 \%$ of all people diagnosed with COVID-19. Based on previous trials we have conducted with NHPs and input from the investigators, we assume $50 \%$ of people will be eligible for this study, and $50 \%$ of those eligible will be interested in participating. This yields an approximate expected recruitment rate of 1-2 participants per day, and an expected recruitment period of 3-6 months.

\section{Study methods \\ Assignment of interventions}

Eligible participants will be randomised using a webbased system developed and maintained by the Ottawa Methods Centre (OMC), an organisation that provides consultation services for areas such as research methodology, biostatistics and data management. Randomisation will occur in permuted blocks of 4 and 6 at ratio of 1:1 to one of the following groups: (1) nutrient therapy with vitamin $\mathrm{D}$, vitamin $\mathrm{C}$, vitamin $\mathrm{K}_{2}$ and zinc or (2) placebo. All study staff, treating physicians and participants will be blinded to the allocation. The staff at Vitazan Professional will be the only personnel with knowledge of treatment allocation to facilitate shipment of study product directly 
to participants. Patients will be randomised once they sign an informed consent form and have their eligibility confirmed by a medical doctor. If unblinding is deemed to be necessary, the trial coordinator will request the treatment allocation from the OMC or Vitazan Professional.

\section{Data collection and management}

Study data will be collected on paper case report forms (CRF) or electronic CRF (eCRF) using the Research Electronic Data Capture (REDCap) platform. All study data, including paper CRFs, eCRFs, and the electronic database, will be managed by the trial coordinator (ML) under the supervision of the principal investigator (DS). Participants will be able to use the REDCap platform to directly enter data or may choose to fill in paper CRFs. To facilitate direct entry to an eCRF, participants will receive an email with a direct link to the eCRF where they can enter their responses. Study data in REDCap will be stored on a secure server located in Toronto, Canada managed by the Canadian College of Naturopathic Medicine (CCNM). Paper CRFs will be kept at the CHI in locked cabinets.

REDCap has been tested to work on Windows 10, the standard software package at the CHI, as well as Mac OS Catalina and above. As required by GCP and Health Canada, the REDCap database has been validated by the study team and is designed to keep track of all users who enter, save, edit, and update data entered to the eCRFs, and to automatically provide a timestamp of all activity. See online supplemental materials section 1.0 for an outline of the data validation plan.

\section{Statistical methods}

All analyses will follow an intention to treat approach. Continuous and quasi-continuous variables (participantreported health \& health status, symptom severity and length of stay) will be compared between arms using unadjusted t-tests. Dichotomous outcomes (delayed return to usual health, hospitalisations and deaths) will be compared between arms using $\chi^{2}$ tests or Fisher's exact tests where appropriate. Time to symptom resolution will be displayed graphically with Kaplan-Meier curves and differences between arms will be compared with a logrank test.

\section{Safety and data monitoring}

External oversight for this trial will be provided by an independent data safety and monitoring board (DSMB). The DSMB will meet either in-person or remotely to discuss matters related to the safety of study participants, validity and integrity of the data, enrollment rate relative to expectations, characteristics of participants, retention of participants, adherence to the protocol and data completeness. The DSMB will review interim data once 100 participants are enrolled or after 4 months, whichever comes first. The DSMB may choose to review data at other times at their discretion. Based on review of the safety data, the DSMB may recommend continuation of the study without modification(s), study interruption, study termination or modification(s) of the trial.

Additionally, the trial will have a quality control monitoring process in place to verify that all data are accurate and complete. Investigators will permit trial-related monitoring, audits and regulatory inspections, and direct access to source data/documents. The monitor will generate a site monitoring report for the qualified investigator detailing significant findings, deviations, deficiencies, plausibility, record completeness and any corrective actions required.

Adverse events (AE) will be collected by the study team for each participant throughout the intervention period plus one additional week using the Common Terminology Criteria for Adverse Events V.5.0. Classic or other symptoms of COVID-19 will not be recorded as AEs. These include fever, new or worsening cough, shortness of breath/difficulty breathing, fatigue, myalgia/ arthralgia, sore throat, sputum production, dysphagia, new olfactory or taste disorders, pneumonia, rhinorrhoea, chills/shakes or nasal congestion. Such events are expected in a population who are COVID-19 positive and are not deemed deviations from the normal course of the disease. Gastrointestinal disturbances (e.g., nausea, vomiting, diarrhoea, upset stomach) are also considered symptoms of COVID-19 but will be reported as AEs as they are possible side effects of the interventions being studied.

\section{Patient and public involvement}

No patients or public persons were involved.

\section{Ethics and dissemination}

This study has received ethical approval from the research ethics boards (REB) of the CCNM and the Ottawa Health Sciences Network (OHSN), as well as regulatory approval from both the Therapeutic Products Directorate (TPD) and Natural and Non-Prescription Health Products Directorate (NNHPD) of Health Canada.

All amendments to the protocol will be reviewed by both REBs and submitted to the NNHPD and the TPD as either a Clinical Trial Notification or Clinical Trial Application Amendment. Both the principal investigator and qualified investigator will sign the approved protocol prior to implementation, and each investigator and member of the research team will be adequately trained prior to carrying out any study-specific tasks after the approval of the amendment.

All participants will sign an informed consent form prior to participating in this study. Clinical staff from COVID-19 test centres will introduce the study to participants and obtain their consent to be contacted by a member of the research team. Study staff will then formally explain all aspects of the trial and answer any questions the patient may have. The patient will be given adequate time to review the consent form and no study activities will take place before the signing of the consent form. 


\section{Privacy and confidentiality}

Participant personal health information will be kept confidential unless release is required by law. Representatives of the OHSN REB, OHRI, CCNM REB, NNHPD or TPD may review original medical records under the supervision of Dr. Seely's staff for audit purposes.

Participants will not be identified in any publications or presentations resulting from this study, unless permission is given by the participant. All paper CRFs will be kept in locked cabinets in a locked office and all databases will be password protected on a secure server. These documents and relevant source documents will be kept for a period of 25 years as required by Health Canada. CRFs will be shredded, and databases will be securely deleted at the end of this retention period.

\section{Clinical relevance}

Regardless of outcome, the results of this and other similar studies will inform the public and the scientific community of the effectiveness of dietary supplements on the overall health of people diagnosed with COVID-19 in the community and their effects on symptom severity and duration. If a positive result is seen, this study could corroborate a safe, affordable treatment option for those suffering from the virus. If a negative outcome is seen, it will help prevent patients from using unproven protection from a natural therapy and paying out of pocket for an ineffective therapy. Although there are several approved vaccines in circulation, their long-term efficacy is unknown. In addition, vaccine access is limited in lowand middle-income countries. For example, the SinoVac COVID-19 vaccine, which is currently undergoing trials in Brazil, ${ }^{33}$ is only $51 \%$ efficacious in preventing symptomatic COVID-19. ${ }^{34}$ Research into potential community treatments of COVID-19 continues to be important and has the potential to contribute to the worldwide public health management of this pandemic and its associated societal burden.

\section{Dissemination and knowledge transfer}

The work done in this study will be disseminated in the form of scientific presentations to complementary, integrative and traditional medical conferences within Canada and internationally. Presentations will be accompanied by published abstracts. The principal mechanism for knowledge transfer will be publication and will include the use of social media as well as press. We will target the most reputable clinical journal for open access publication due to the potential impact of this investigation.

\section{Administrative information}

\section{Protocol revision chronology}

Protocol revision chronology can be found in online supplemental materials, section 2.0.

\footnotetext{
Author affiliations

${ }^{1}$ Research, The Centre for Health Innovation, Ottawa, Ontario, Canada

${ }^{2}$ Clinical Epidemiology, Ottawa Hospital Research Institute, Ottawa, Ontario, Canada

${ }^{3}$ Research, Canadian College of Naturopathic Medicine, Toronto, Ontario, Canada
}

${ }^{4}$ Department of Pharmacy, The Ottawa Hospital, Ottawa, Ontario, Canada

Contributors DS conceived of the study. KW, DS, ML, EC and AP conducted background review and created the study design. ML was responsible for ethical and regulatory submissions. DAF provided methodological expertise and input regarding outcome selection. TR provided statistical expertise and helped formulate the statistical analysis plan. SK provided expertise in determining potential pharmacological interactions with the investigational products. All authors contributed to refinement of the protocol and approved this manuscript.

Funding Funding for this study was provided by the Ottawa Integrative Cancer Centre Foundation and private support from Mavis and Martin Sacher. Investigational product for this study was provided in-kind by New Roots Herbal under the brand name Vitazan Professional.

Disclaimer The funder had minimal input with regards to the investigational products used in this study. The sponsor did not have any role in the study design. Both parties will not have any role during the study with regards to its execution, analyses, interpretation of the data, or decision to submit for publication.

Competing interests None declared.

Patient consent for publication Not applicable.

Provenance and peer review Not commissioned; externally peer reviewed.

Data availability statement Not applicable.

Supplemental material This content has been supplied by the author(s). It has not been vetted by BMJ Publishing Group Limited (BMJ) and may not have been peer-reviewed. Any opinions or recommendations discussed are solely those of the author(s) and are not endorsed by BMJ. BMJ disclaims all liability and responsibility arising from any reliance placed on the content. Where the content includes any translated material, BMJ does not warrant the accuracy and reliability of the translations (including but not limited to local regulations, clinical guidelines, terminology, drug names and drug dosages), and is not responsible for any error and/or omissions arising from translation and adaptation or otherwise.

Open access This is an open access article distributed in accordance with the Creative Commons Attribution Non Commercial (CC BY-NC 4.0) license, which permits others to distribute, remix, adapt, build upon this work non-commercially, and license their derivative works on different terms, provided the original work is properly cited, appropriate credit is given, any changes made indicated, and the use is non-commercial. See: http://creativecommons.org/licenses/by-nc/4.0/.

\section{ORCID iDs}

Mark Legacy http://orcid.org/0000-0003-4417-1656

Tim Ramsay http://orcid.org/0000-0001-8478-8170

Dean A Fergusson http://orcid.org/0000-0002-3389-2485

\section{REFERENCES}

1 Pascarella G, Strumia A, Piliego C, et al. COVID-19 diagnosis and management: a comprehensive review. J Intern Med 2020;288:192-206.

2 World Health Organization. Weekly Epidemiological Update on COVID-19 - Edition 452021.

3 Health Canada. Approved COVID-19 vaccines, 2021. Available: https://www.canada.ca/en/health-canada/services/drugs-healthproducts/covid19-industry/drugs-vaccines-treatments/vaccines.html [Accessed 24 Jun 2021].

4 Polack FP, Thomas SJ, Kitchin N. Safety and efficacy of the BNT162b2 mRNA Covid-19 vaccine. N Engl J Med 2020;384:1576-7.

5 Frederiksen LSF, Zhang Y, Foged C. The long road toward COVID-19 herd immunity: vaccine platform technologies and mass immunization strategies. Front Immunol 2020;1817:11.

6 Health Canada. COVID-19 treatments 2021.

7 Wang Y, Zhang D, Du G, et al. Remdesivir in adults with severe COVID-19: a randomised, double-blind, placebo-controlled, multicentre trial. Lancet 2020;395:1569-78.

8 Beigel JH, Tomashek KM, Dodd LE. Remdesivir for the Treatment of Covid-19 - Preliminary Report. N Engl J Med 2020.

9 Health Canada. Bamlanivimab for injection 2021.

10 Health Canada. Product Monograph - Casirivimab and Imdevimab for Injection 2021.

11 Michienzi SM, Badowski ME. Can vitamins and/or supplements provide hope against coronavirus? Drugs Context 2020;9. doi:10.7573/dic.2020-5-7. [Epub ahead of print: 2206 2020]. 
12 Beigmohammadi MT, Bitarafan S, Hoseindokht A, et al. Impact of vitamins $A, B, C, D$, and $E$ supplementation on improvement and mortality rate in ICU patients with coronavirus-19: a structured summary of a study protocol for a randomized controlled trial. Trials 2020;21:614.

13 Hu X-Y, Wu R-H, Logue M, et al. Andrographis paniculata (Chuān Xīn Lián) for symptomatic relief of acute respiratory tract infections in adults and children: a systematic review and meta-analysis. PLoS One 2017;12:e0181780.

14 Ding Y, Chen L, Wu W, et al. Andrographolide inhibits influenza A virus-induced inflammation in a murine model through NF- $\kappa B$ and JAK-STAT signaling pathway. Microbes Infect 2017;19:605-15.

15 Heinz SA, Henson DA, Austin MD, et al. Quercetin supplementation and upper respiratory tract infection: a randomized community clinical trial. Pharmacol Res 2010;62:237-42.

16 Pitt HA, Costrini AM. Vitamin C prophylaxis in marine recruits. JAMA 1979;241:908-11.

17 Hemilä H. Vitamin C and infections. Nutrients 2017;9. doi:10.3390/ nu9040339. [Epub ahead of print: 29 Mar 2017].

18 Hemilä $\mathrm{H}$, Chalker E. Vitamin $\mathrm{C}$ for preventing and treating the common cold. Cochrane Database Syst Rev 2013;1:Cd000980.

19 Martineau AR, Jolliffe DA, Hooper RL, et al. Vitamin D supplementation to prevent acute respiratory tract infections: systematic review and meta-analysis of individual participant data. BMJ 2017;356:i6583.

20 Shimizu Y, Ito Y, Yui K, et al. Intake of 25-hydroxyvitamin D3 reduces duration and severity of upper respiratory tract infection: a randomized, double-blind, placebo-controlled, parallel group comparison study. J Nutr Health Aging 2018;22:491-500

21 Jung $\mathrm{H}$, Seo M-W, Lee S, et al. Vitamin D3 supplementation reduces the symptoms of upper respiratory tract infection during winter training in vitamin D-Insufficient Taekwondo athletes: a randomized controlled trial. Int J Environ Res Public Health 2018;15:2003.

22 Science M, Johnstone J, Roth DE, et al. Zinc for the treatment of the common cold: a systematic review and meta-analysis of randomized controlled trials. CMAJ 2012;184:E551-61.

23 Lau FH, Majumder R, Torabi R. Vitamin D insufficiency is prevalent in severe COVID-19. medRxiv 2020.

24 Ohaegbulam KC, Swalih M, Patel P, et al. Vitamin D supplementation in COVID-19 patients: a clinical case series. Am J Ther 2020;27:e485-90.
25 Dofferhoff ASM, Piscaer I, Schurgers LJ. Reduced vitamin K status as a potentially modifiable prognostic risk factor for COVID-19. Clin Infect Dis 2021;73:e4039-404.

26 Pal R, Banerjee M, Bhadada SK, et al. Vitamin D supplementation and clinical outcomes in COVID-19: a systematic review and metaanalysis. J Endocrinol Invest 2022;45:53-68.

27 Thomas S, Patel D, Bittel B, et al. Effect of high-dose zinc and ascorbic acid supplementation vs usual care on symptom length and reduction among ambulatory patients with SARS-CoV-2 infection: the COVID A to $Z$ randomized clinical trial. JAMA Netw Open 2021;4:e210369.

28 Wiersinga WJ, Rhodes A, Cheng AC, et al. Pathophysiology, transmission, diagnosis, and treatment of coronavirus disease 2019 (COVID-19). JAMA 2020;324:782.

29 EuroQol Group. EuroQol--a new facility for the measurement of health-related quality of life. Health Policy 1990;16:199-208.

30 Tenforde MW, Kim SS, Lindsell CJ, et al. Symptom Duration and Risk Factors for Delayed Return to Usual Health Among Outpatients with COVID-19 in a Multistate Health Care Systems Network United States, March-June 2020. MMWR Morb Mortal Wkly Rep 2020;69:993-8.

31 National Institute for Health and Care Excellence. COVID-19 rapid guideline: managing the long-term effects of COVID-19, 2020. Available: https://www.nice.org.uk/guidance/ng188 [Accessed 3 Jan 2021].

32 US Food and Drug Admiinistration. Assessing COVID-19RelatedSymptoms in outpatient adult and adolescent subjects in clinical trials of drugs and biological products for COVID-19 prevention or treatment: guidance for industry, 2020. Available: https://www.fda.gov/media/142143/download

33 Palacios R, Patiño EG, de Oliveira Piorelli R, et al. Double-Blind, Randomized, Placebo-Controlled Phase III Clinical Trial to Evaluate the Efficacy and Safety of treating Healthcare Professionals with the Adsorbed COVID-19 (Inactivated) Vaccine Manufactured by Sinovac - PROFISCOV: A structured summary of a study protocol for a randomised controlled trial. Trials 2020;21:853.

34 World Health Organization. Interim recommendations for use of the inactivated COVID-19 vaccine, CoronaVac, developed by Sinovac, 2020. 